\title{
Experiencia colombiana en reparación de núcleos de transformadores de potencia
}

\author{
Juan Carlos Castro Galeano* \\ César Alejandro Rincón Becerra* \\ Luis Enrique Echavarría Cuartas ${ }^{* * *}$
}

Recibido: 05/02/2014 • Aceptado: 15/05/2015

\section{Resumen}

Este artículo presenta el reporte de caso sobre fallas presentadas en el núcleo de transformadores de potencia y algunas experiencias técnicas y metodológicas en la reparación parcial y total de los mismos a varias unidades, hechas en Industrias Explorer Ingeniería S. A. S., empresa dedicada al mantenimiento y reparación de transformadores. También es presentada la metodología para la selección del tipo de lámina, sistema de corte, ensamble, ajuste y prensado del núcleo, ya que estas actividades son decisivas para conseguir un equipo con menores pérdidas y corrientes de vacío, así como menores niveles de ruido.

Se describen las etapas para cálculo del flujo de operación del núcleo, circuito de prueba para la saturación del mismo, consideraciones para realizar la inspección termográfica y medición de las pérdidas de vacío, selección del tipo de lámina y técnicas de ensamble empleadas.

Se presentan algunas experiencias como: cambio de medio núcleo, reaislamiento de zonas afectadas empleando fibras Nómex entre láminas, cambio total del núcleo por corte mal realizado desde fábrica, cambio total del núcleo por doble aterrizamiento que ocasionó calentamiento del mismo y afectó el aislamiento de sus láminas dejándolas en corto. En todos los casos se evidencia una disminución de las pérdidas de vacío. Finalmente se presentan el comportamiento de los transformadores después de ser puestos nuevamente en servicio.

Palabras clave: magnetización, núcleo, termografía, transformador de potencia.

Ingeniero electricista, docente Ingeniería Electromecánica, Grupo de Investigación GridsE. Universidad Pedagógica y Tecnológica de Colombia, Facultad Seccional Duitama. Calle 22 carrera 18. Teléfono 3124801866. Correo electrónico juan.castrogaleano@uptc.edu.co

** Ingeniero electricista. Gerente General Industrias Explorer, Av. De las Américas 20-60. Duitama Boyacá. Correo electrónico: gerencia@industriasexplorer.com

*** Profesional técnico. Área Mantenimiento Transmisión. Subestación Colombia. Empresas Públicas de Medellín EPM. Correo electrónico luis.echavarria.cuartas@epm.com.co 


\title{
Colombian experience in power transformers core repair
}

\begin{abstract}
This article presents a case report about faults presented in power transformer cores and shows some technical and methodological experiences in partial and total repair to several units, carried out in Industrias Explorer Ingeniería S.A.S, a company dedicated to the maintenance and repair of power transformers. The methodology for the selection of foil type, court system, assemble, adjustment and pressing of the core are also presented, since these activities are crucial to get an equipment with fewer no-load losses, as well as lower levels of noise.

The stages for the magnetic flux density calculation, test circuit for core saturation, considerations to thermographic inspection and no-load losses measurement, type foil selection and assembling technique employed are described.

Some experiences are presented: partial core reposition, replacement core isolation of affected areas using Nomex fibers between sheets, total core replacement due to failure caused by poor cutting during manufacturing process, replacement of total core due to failure caused by double grounding that produced heating of it affecting the interlamination isolation between their sheets placing them in shortcut. In all cases there is a reduction on no-load losses. Finally the performance of power transformers after been in service is presented and described.
\end{abstract}

Key words: Core, magnetization, power transformer, thermography. 


\section{INTRODUCCIÓN}

El transformador es una máquina eléctrica estática que transfiere energía de un circuito a otro por medio de un campo magnético común. Cuando una corriente eléctrica alterna fluye a través de un conductor, se crea un campo magnético alrededor del mismo, si un segundo conductor es puesto en este campo de tal forma que las líneas de flujo lo enlacen, es inducido un voltaje en terminales del segundo conductor. El principio de funcionamiento del transformador se basa en el uso de un flujo magnético producido por una bobina para inducir voltaje en otra bobina. Si un transformador fuera construido con núcleo de aire entre sus devanados, este sería ineficiente debido a que el porcentaje del flujo del primer devanado que enlaza el segundo sería mínimo, y por tanto, el voltaje inducido en el segundo devanado, pequeño.

Para incrementar el voltaje de salida puede ser aumentado el número de espiras o incrementada la cantidad de flujo que enlaza el segundo devanado. Aumentando el número de espiras se incrementan los costos significativamente, mientras que incrementando el flujo del primer devanado que enlaza el segundo se obtiene una máquina mucho más eficiente. El aumento del flujo que enlaza los devanados se logra empleando un núcleo de hierro, acero o acero al silicio, el cual tiene una propiedad mucho mayor para transportar el flujo magnético que el aire.

Los transformadores pertenecen a los aparatos más eficientes y confiables de los sistemas de potencia; su factor de eficiencia es extremadamente alto, con valores cercanos al 99,5\%. Sin embargo, las pérdidas en el núcleo de un transformador permanecen 24 horas al día, es decir, también en el estado sin carga. Durante la fabricación, reparación y repotenciación de transformadores de potencia, actualmente se emplea acero al silicio de grano altamente orientado, el cual tiene bajas pérdidas y magnetostricción pequeña pero finita, con lo cual se logra disminuir las pérdidas de vacío y el ruido audible directamente relacionado con el núcleo, aumentando la conciencia energética y el cuidado del medio ambiente al disminuir el consumo de energía.

El núcleo del transformador de potencia es el componente de mayor volumen y su función de acoplar magnéticamente los devanados está asociada con la densidad de flujo magnético en que este puede operar, valor que, a su vez, depende de la reluctancia, tipo de lámina, espesor, tipo de cierre, apilamiento y prensado. Un transformador con núcleo de menor reluctancia es más eficiente y presenta menor corriente de vacío. En condiciones normales de operación, el núcleo se encuentra aislado eléctricamente de su sistema de prensado, y las láminas, aisladas entre sí. Cuando estos aislamientos están mal diseñados, deteriorados o mal ensamblados, se producen pequeños arcos entre láminas o yugos y prensas, los cuales empiezan a producir puntos calientes colocando parte de las láminas en cortocircuito; a su vez, se incrementan las corrientes circulantes 
de Eddy y la temperatura de operación del mismo, generando gases combustibles en el aceite y acelerando su degradación, lo que conlleva a una disminución de la vida útil del transformador, de allí la importancia de un núcleo en buen estado.

\section{MATERIALES Y MÉTODOS}

\subsection{Función del núcleo en el transformador}

La característica para conducir el flujo magnético es llamada permeabilidad y es mucho mayor en el hierro y en el acero que en el aire. Los aceros modernos tienen permeabilidades relativas del orden de algunas decenas de millar comparadas con 1,0 para el aire; esto significa que la propiedad del núcleo de acero para transportar el flujo magnético es 1500 veces la del aire. Los núcleos de los transformadores son fabricados utilizando chapas magnéticas de acero al silicio de grano orientado de bajas pérdidas con espesores entre 0,23 y $0,30 \mathrm{~mm}$, laminadas en frío y aisladas en ambas caras con silicato-fosfato de magnesio (Carlite). Los cortes se realizan a $45^{\circ}$, lo cual minimiza el camino del flujo magnético en dirección no coincidente con la laminación de las chapas.

La mayoría de transformadores se ensambla apilando el núcleo en columnas (core type). Con el propósito de reducir la corriente de vacío, lograr mejor desempeño magnético, menores pérdidas, bajos niveles de ruido y calentamiento en el núcleo, el ensamble de este se realiza trabando las chapas entre sí, en forma de un escalonado progresivo, utilizando la técnica conocida como "step-lap". Durante los años 80 y 90 se empleaban las uniones de traslape sencillo SSL, pero hoy en día este sistema fue reemplazado por uniones con traslapes múltiples MSL para disminuir las pérdidas de vacío, la magnetostricción y el ruido audible.

Las regiones de unión son las "regiones más calientes" en el núcleo. Como mencionan [1] y [2], muchos parámetros geométricos están involucrados en la configuración de la unión, tales como la longitud del entrehierro de aire $g$, la longitud del traslape $a$, cantidad de láminas por paquete $s$ y en especial el número $N$ de pasos en las regiones del traslape, como se aprecia en la figura 1 .

Aunque los núcleos de los transformadores son apilados cuidadosamente, aparecen en las uniones algunos entrehierros de aire inevitables de longitud $\mathrm{g}$, incrementando su reluctancia magnética. La figura 2 ilustra la bien conocida distribución básica del flujo. Para evitar el camino de alta reluctancia del entrehierro, el flujo escapa a las laminaciones vecinas las cuales actúan como un entrehierro puente (GB) y se tornan sobrecargadas. En los finales de la lámina el flujo $\Phi z$ surge en dirección normal. La transferencia del flujo en las láminas vecinas muestra una distribución local la cual depende fuertemente de B, como se discute en [3]. 


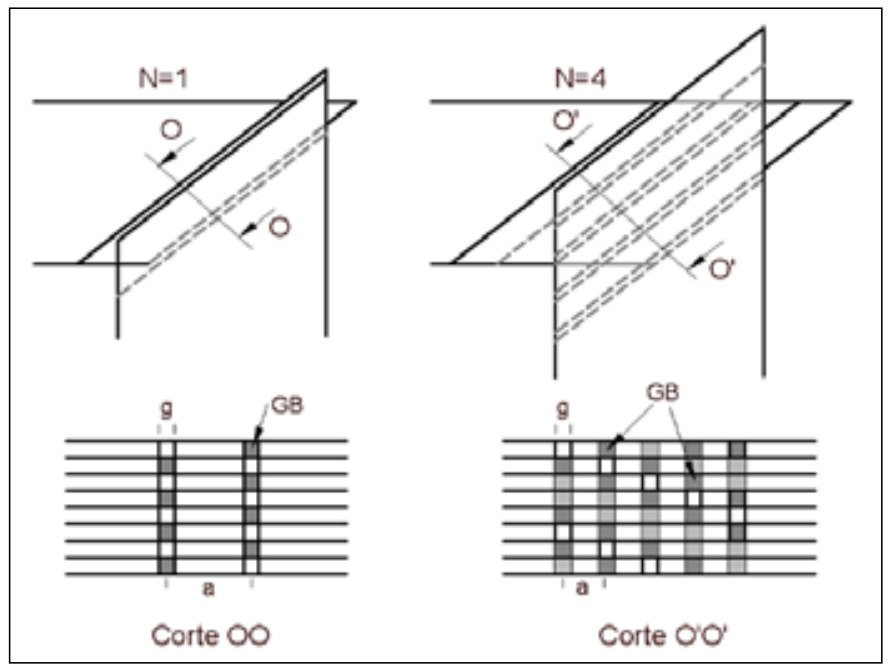

Figura 1. Diferentes diseños de uniones: a) SSL-Traslape sencillo $(\mathrm{N}=1)$;

b) MSL- Traslape múltiple $(\mathrm{N}=4)$.

Fuente: elaboración propia
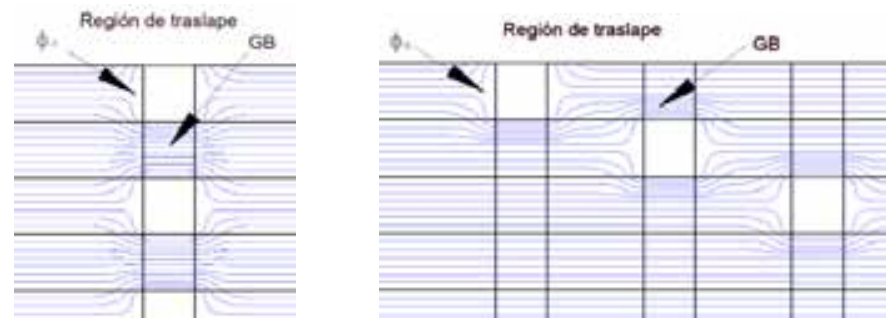

Figura 2. Uniones y regiones en el traslape del núcleo

Fuente: elaboración propia

En las uniones multi step-lap el número de entrehierros es incrementado, por tanto la concentración de flujo en el área de unión en el entrehierro puente GB es reducida, como se ve en las figuras 3 y 4 .

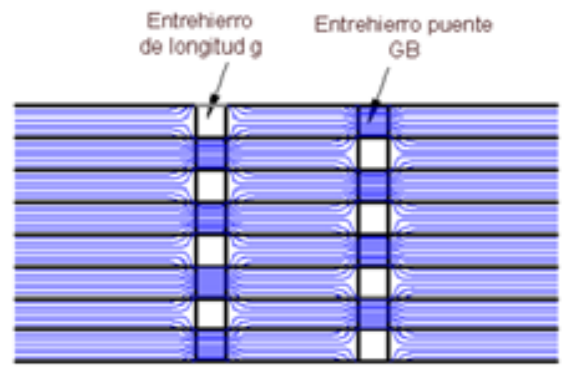

Figura 3. Concentración del flujo en el entrehierro puente GB (Mayor en SSL)

Fuente: elaboración propia 


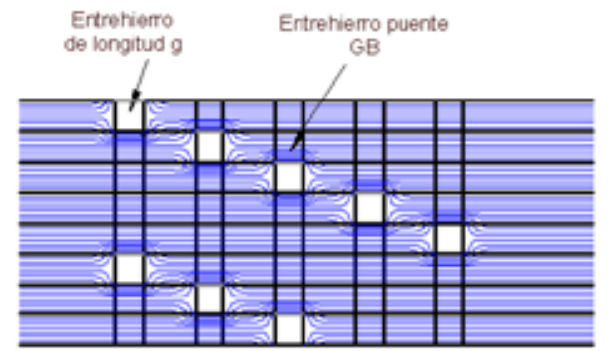

Figura 4. Concentración del flujo en el entrehierro puente GB (Menor en MSL) Fuente: elaboración propia

Normalmente se emplean cinco pasos $\mathrm{N}$ por libro de láminas y dos láminas por paso, como indican las figuras 5 y 6 [4], [5].

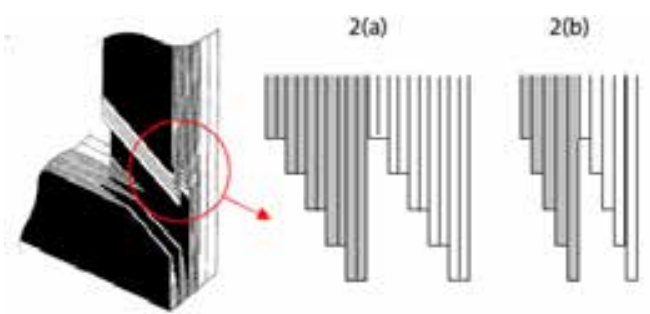

Figura 5. Multi Step Lap cinco pasos por libro a) dos láminas por paso, b) una lámina por paso. Fuente: elaboración propia

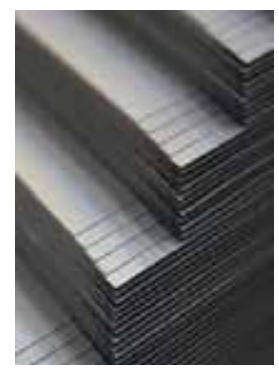

Figura 6. Multi Step Lap cinco pasos por libro Fuente: elaboración propia

\subsection{DIAGNÓSTICO INICIAL DE FALLAS EN EL NÚCLEO}

Una de las técnicas más útiles y usadas para el análisis de condición consiste en el análisis de los gases disueltos en el aceite. Esta técnica es muy sensible en un amplio rango para encontrar una operación inadecuada térmica o eléctrica. De acuerdo con los estándares ASTM D-3613, ASTM D-3612 y ANSI IEEE C57.104, el hidrógeno y los gases hidrocarbonos son generados debido al rompimiento térmico del aceite mi- 
neral indicando en ciertas relaciones la severidad del sobrecalentamiento. La presencia de poco acetileno y un amplio espectro de otros gases, usualmente es el resultado de partes metálicas sobrecalentadas por concentraciones de flujo o corrientes circulantes, sin celulosa presente, indicando láminas del núcleo en cortocircuito, cortos entre el núcleo y prensas o partes metálicas.

De acuerdo con el criterio del gas clave y Rogers [6], las relaciones acetileno/ etileno, metano/hidrógeno y etileno/etano indican sobrecalentamiento localizado en el núcleo debido a concentraciones de flujo, incremento de temperatura de puntos calientes y/o sobrecalentamiento en el cobre debido a falsos contactos (formación de carbón) como presenta la tabla 1.

Tabla 1: Criterio de las razones de Rogers

\begin{tabular}{|l|c|c|c|}
\hline \multicolumn{1}{|c|}{ Falla térmica } & $\mathrm{C}_{2} \mathrm{H}_{2} / \mathrm{C}_{2} \mathrm{H}_{4}$ & $\mathrm{CH}_{4} / \mathrm{H}_{2}$ & $\mathrm{C}_{2} \mathrm{H}_{4} / \mathrm{C}_{2} \mathrm{H}_{6}$ \\
\hline $150{ }^{\circ} \mathrm{C}$ a $300^{\circ} \mathrm{C}$ & 0 & 2 & 0 \\
\hline $300^{\circ} \mathrm{C} \mathrm{a} 700^{\circ} \mathrm{C}$ & 0 & 2 & 1 \\
\hline$>700^{\circ} \mathrm{C}$ & 0 & 2 & 2 \\
\hline
\end{tabular}

Fuente: elaboración propia

Después de analizar los datos históricos de cromatografía de gases, cuando se sospecha del núcleo y se decide sacar de servicio el transformador, este es llevado a planta para ser desencubado, retirar sus bobinas y hacer posteriormente una inspección visual detallada al núcleo y sus láminas. Seguidamente se cierra el yugo y se realiza la prueba de magnetización e inspección termografía.

\subsection{CÁLCULO DE LA DENSIDAD DE FLUJO DE OPERACIÓN}

La densidad de flujo de operación del núcleo de los transformadores de potencia se encuentra entre 14000 y 18000 Gauss; esto depende del año de fabricación, diseño, fabricante, pérdidas garantizadas, entre otros. Empleando la expresión (1) se establece el valor más aproximado para el cálculo de un circuito magnético, [7].

$$
\beta=\frac{V_{B O B I N A}}{4,44 \times f \times A_{i} \times \rho \times N \times 1 \times 10^{-8}}
$$

\section{Donde,}

ß: Densidad de flujo de operación del núcleo, Gauss.

f: Frecuencia, $\mathrm{Hz}$. 
Ai : Sección transversal del núcleo, $\mathrm{mm}^{2}$. Valor medible en el núcleo.

$\rho$ : Factor de apilamiento, valor asumido de 0,94

$N$ : Número de espiras. Posible de contar en alguna de las bobinas.

$V_{\text {BOBINA }}=$ Voltaje de la bobina, $V_{F F}$ para conexión delta o $V_{F N}$ para conexión $Y$.

La densidad de flujo de operación puede ser calculada por cualquier devanado; el número de espiras y la tensión de la bobina deben ser correspondientes al devanado considerado.

Una vez conocido el flujo de operación del núcleo, para determinar el número de espiras a emplear en la prueba de laboratorio se considera el voltaje de la bobina como el máximo suministrado por el generador empleado para las pruebas y se despeja $\mathrm{N}$ de la expresión (1).

Cuando no es posible contar el número exacto de espiras, se arrolla una bobina con un número de espiras conocido en cada columna del núcleo. Seguidamente este bobinado es energizado gradualmente mientras se registra la lectura de corriente. $\mathrm{Si}$ se alcanza la tensión máxima del generador y la corriente no crece significativamente es necesario retirar una espira, o si la corriente aumenta con poca tensión, es necesario arrollar una espira. Se grafica la curva de voltaje contra corriente, y con el número de espiras encontrado y la tensión alcanzada en el codo de saturación se emplea la expresión (1) para calcular la densidad de flujo.

\subsection{PRUEBA DEL AISLAMIENTO DE LAS LÁMINAS}

Las láminas están provistas de una fina capa de aislamiento inorgánico basada en silicato-fosfato de magnesio (carlite) por ambas caras para aumentar la resistencia entre láminas y reducir las pérdidas por corrientes de Eddy. Dicho aislamiento se aplica durante el proceso de templado de la lámina, su espesor se encuentra entre $2 \mu \mathrm{m}-5$ $\mu \mathrm{m}$ y la resistencia de aislamiento de la superficie es mayor a $10 \Omega / \mathrm{cm}^{2}$, [8] y [9].

Durante el ensamble del núcleo, se verifica el recubrimiento aislante de las láminas, empleando el procedimiento sugerido por el estándar internacional [10]; dado que no existe un criterio normalizado del valor del aislamiento, se emplea el dado en [2], el cual sugiere que al menos el $80 \%$ de las lecturas realizadas sobre una muestra de núcleo debe ser mayor de $2 \Omega$ y el $50 \%$ mayor de $5 \Omega$. Para ello se diseñó y construyó un dispositivo el cual consta de dos electrodos planos con una diferencia de potencial de 1,5 VDC entre ellos; dichos electrodos se ubican a lo largo de las láminas y entre láminas adyacentes. Cuando el recubrimiento aislante está defectuoso, se enciende un piloto de señalización (ver figuras 7 y 8). 


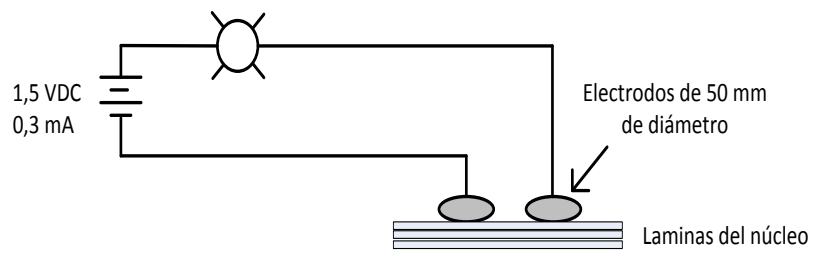

Figura 7. Circuito dispositivo para verificar aislamiento de las láminas del núcleo Fuente: elaboración propia

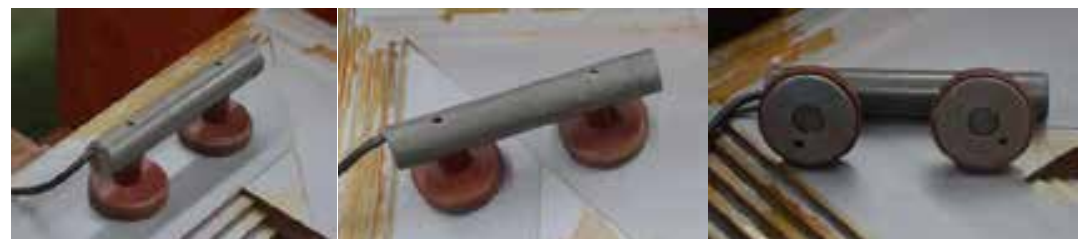

Figura 8. Electrodos para verificar aislamiento de las láminas del núcleo. Fuente: elaboración propia

\subsection{PRUEBA DE AISLAMIENTO NÚCLEO -PRENSA}

Una vez finalizado el ensamble del núcleo, se verifica el aislamiento núcleo contra tierra, energizando el núcleo y aterrizando la prensa (ver figura 9). El voltaje de prueba máximo es de $1000 \mathrm{~V}$.

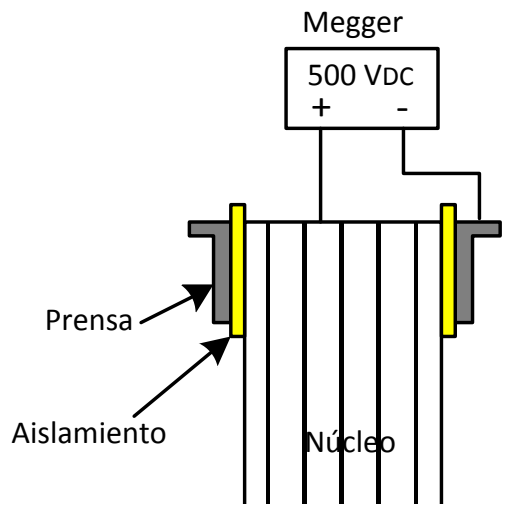

Figura 9. Prueba de aislamiento núcleo-prensa Fuente: elaboración propia

La resistencia de aislamiento en núcleos usados se encuentra en el rango de 100 a $400 \mathrm{M} \Omega$ y en núcleos nuevos mayores a $500 \mathrm{M} \Omega$; en ambos casos el índice de polarización $I P$ debe ser mayor de 1,0 de acuerdo con [11]. Valores menores a 100 $\mathrm{M} \Omega$ indican deterioro del aislamiento, y menores a $10 \mathrm{M} \Omega$, que existirán corrientes circulantes entre núcleo y prensa, lo cual debe ser investigado. En la tabla 2 se presenta el caso de una prueba a un núcleo nuevo. 
Tabla 2: Ejemplo de resistencia de aislamiento núcleo-prensa.

\begin{tabular}{|c|c|c|c|c|c|c|c|c|}
\hline \multicolumn{7}{|c|}{$\begin{array}{c}\text { Resistencia de aislamiento } \\
\text { núcleo-prensa, }[G \Omega]\end{array}$} & \multirow{3}{*}{ R.A. } & \multirow{3}{*}{ I.P. } \\
\hline \multicolumn{7}{|c|}{ Tiempo de prueba, $\quad$ [min] } & & \\
\hline 0,5 & 1 & 2 & 3 & 5 & 8 & 10 & & \\
\hline 62 & 73 & 79 & 82 & 82 & 80 & 79 & 1,2 & 1,1 \\
\hline
\end{tabular}

Fuente: elaboración propia

El núcleo es aterrizado en solo un punto; algunos núcleos disponen de un buje para realizar esta conexión externamente y poder así verificar su aislamiento durante las pruebas eléctricas de diagnóstico del transformador.

\section{RESULTADOS Y DISCUSIÓN}

\subsection{Caso 1: Autotransformador monofásico 45/60 MVA, 220 / 110 / 46 kV}

Desde 1999 hasta 2009 el transformador presentaba aumento en los gases etano, metano, monóxido y etileno, siendo este último el más crítico. En enero de 2010 el transformador presentó falla en la bobina de $46 \mathrm{kV}$. El transformador fue llevado al taller de reparación y una vez retiradas las bobinas y fibras de aislamiento de las columnas, adicional a la falla en el devanado, se evidenciaron pequeños cortos entre láminas en todas las columnas, como presentan las figuras 10 y 11.

Se hizo la prueba de magnetización e inspección termográfica y se encontraron numerosos puntos calientes entre los fletajes lo que indica la existencia de otros puntos calientes no detectados en la inspección visual (ver figuras 12 y 13).

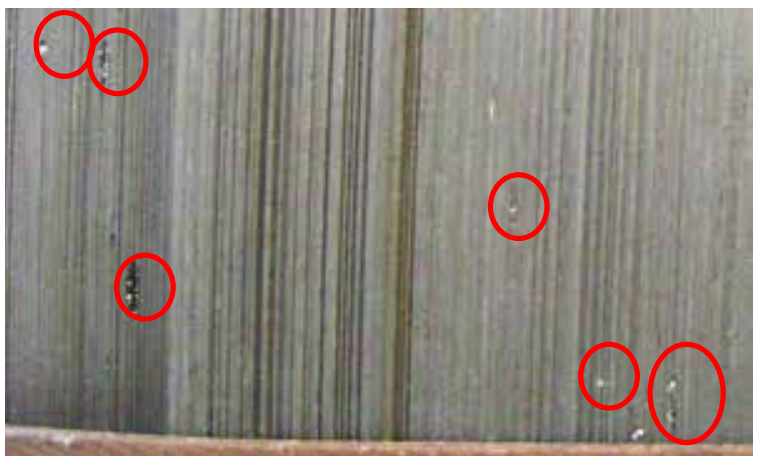

Figura 10. Pequeños cortos en el núcleo.

Fuente: elaboración propia 


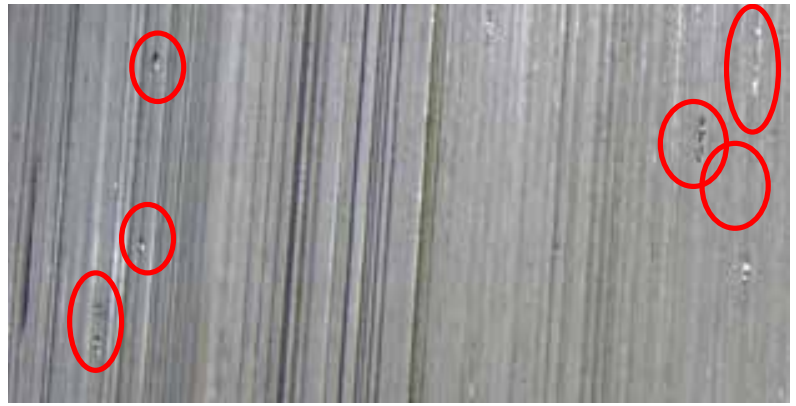

Figura 11. Pequeños cortos en el núcleo.

Fuente: elaboración propia

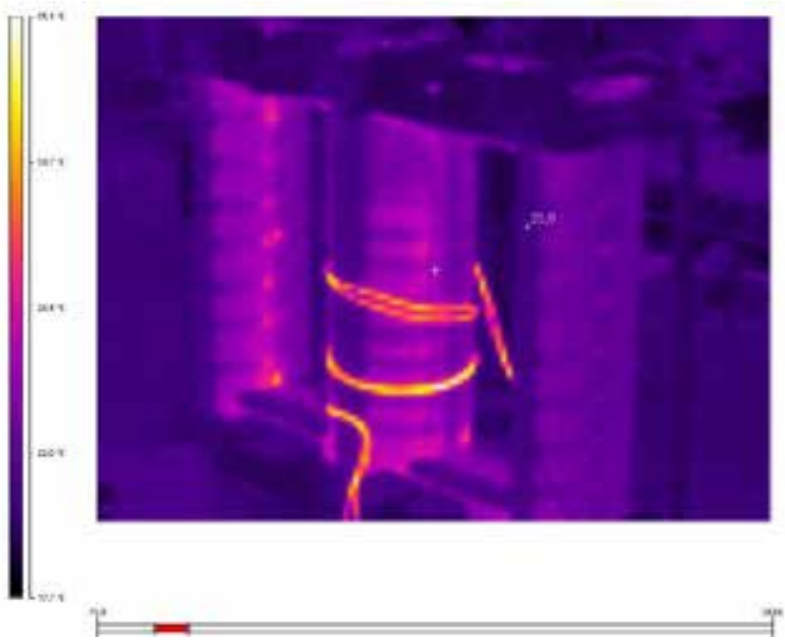

Figura 12. Termografía general. (Puntos calientes debajo de los fletajes) Fuente: elaboración propia

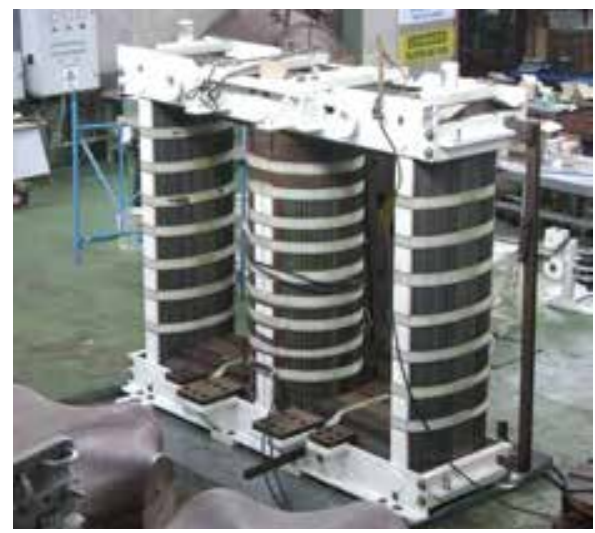

Figura 13. Vista general del núcleo Fuente: elaboración propia 
Los cortos y puntos calientes encontrados fueron reparados parcialmente abriendo cuidadosamente las láminas e instalando fibras Nómex de $0,1 \mathrm{~mm}$ entre ellas. Los fletajes fueron cambiados y se barnizó todo el núcleo, como se ve en las figuras 14, 15 y 16.

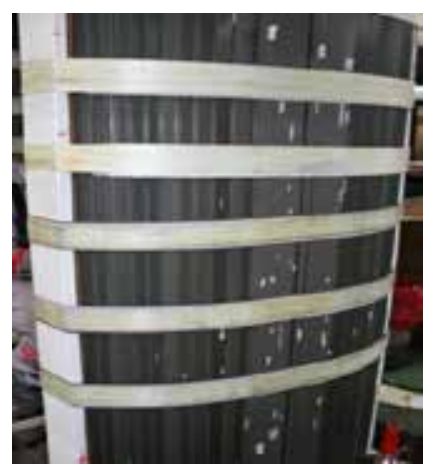

Figura 14. Laminas del núcleo con fibras Nómex entre láminas Fuente: elaboración propia

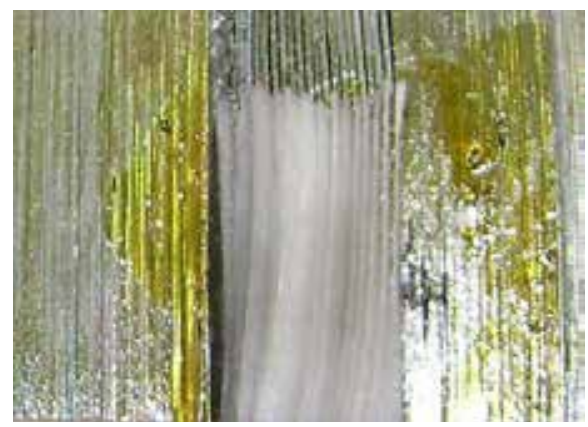

Figura 15. Detalle reparación parcial Fuente: elaboración propia

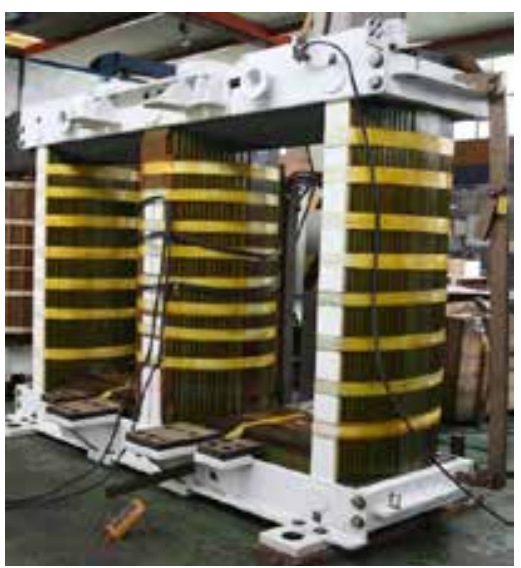

Figura 16. Núcleo con reparaciones parciales y barnizado Fuente: elaboración propia 
Después de este proceso, se repitió la prueba de magnetización del núcleo y no se encontraron puntos calientes, como se aprecia en la figura 17.

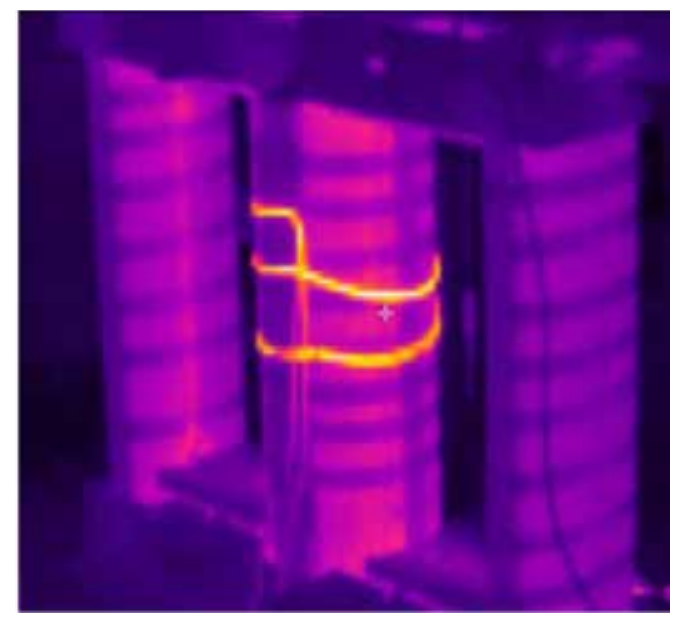

Figura 17. Termografía final sin puntos calientes Fuente: elaboración propia

En abril de 2011, una vez el transformador fue reparado y puesto en servicio nuevamente, fue monitorizado mediante cromatografía de gases, y se encontró un aumento en etano, metano, monóxido y etileno. Aunque dicho aumento fue en menor proporción al anterior, se sospechó de nuevos cortos en el núcleo; por consiguiente, el transformador fue desenergizado, retirado el aceite y realizada una inspección visual interna que confirmó la sospecha.

Se concluyó que los cortos en el núcleo de esta unidad fueron el resultado del mal corte de las láminas durante el proceso se fabricación; cortos que con el tiempo evolucionaron produciendo pequeñas masas similares a puntos de soldaduras, que calentaron el núcleo y generando gases.

En junio de 2012 el transformador fue retirado de servicio y se realizó el cambio total del núcleo, en diciembre de 2012 fue puesto nuevamente en servicio, y a la fecha los gases se encuentran estables.

El costo de reparación de este equipo fue de \$ USD 400.000; cerca del $44 \%$ del valor de reposición a nuevo que es de \$USD 900.000, aproximadamente, incluyendo el cambio total del núcleo \$ USD 100.000, $11 \%$ del valor del equipo. Estas cifras demuestran que la reparación fue muy rentable. Adicionalmente, las pérdidas de vacío presentaron una disminución de $5 \mathrm{~kW}$, representando un ahorro cercano a los USD 100.000 en un periodo de 20 años. 


\subsection{Caso 2: Transformador 104,5 MVA, $220 / 13,8$ kV}

Este transformador presentaba incremento notorio en los gases etano, metano, monóxido y etileno. Había sido reparado en dos ocasiones y en una de ellas el núcleo quedó aterrizado en dos partes por lo que entre sus láminas se presentó circulación de corrientes circulantes que las sobrecalentaron y quemaron su recubrimiento aislante, quedando la gran mayoría en cortocircuito.

En la inspección termográfica inicial se encontró un sobrecalentamiento en la unión del yugo superior con la columna central que alcanzó $102^{\circ} \mathrm{C}$ y puntos calientes localizados en los traslapes y mostró corto entre láminas (ver figuras 18 a 21).

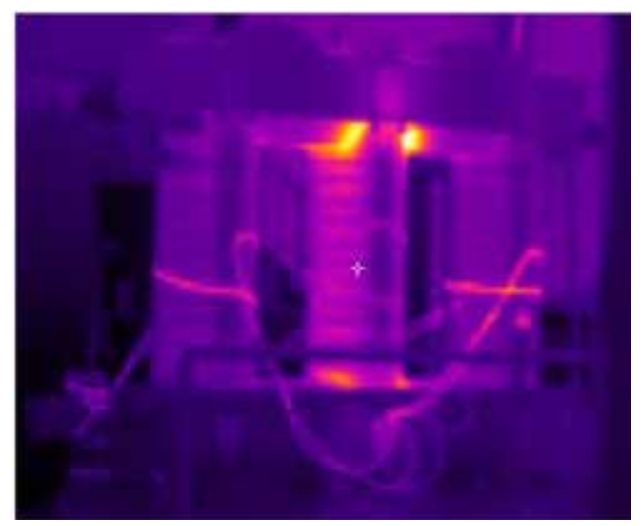

Figura 18. Termografía general núcleo original Fuente: elaboración propia

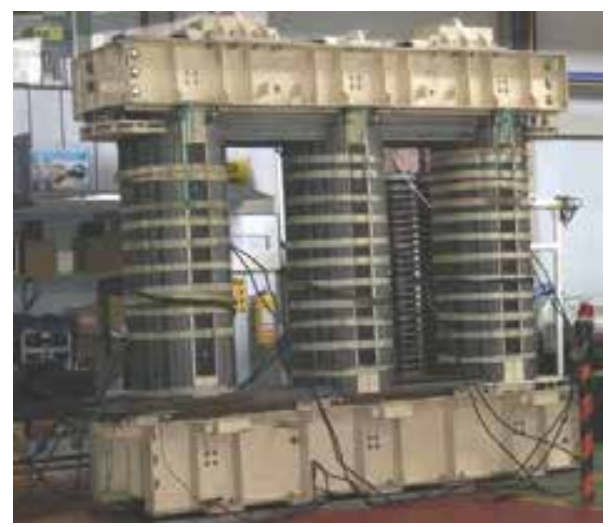

Figura 19. Termografía general núcleo original Fuente: elaboración propia 


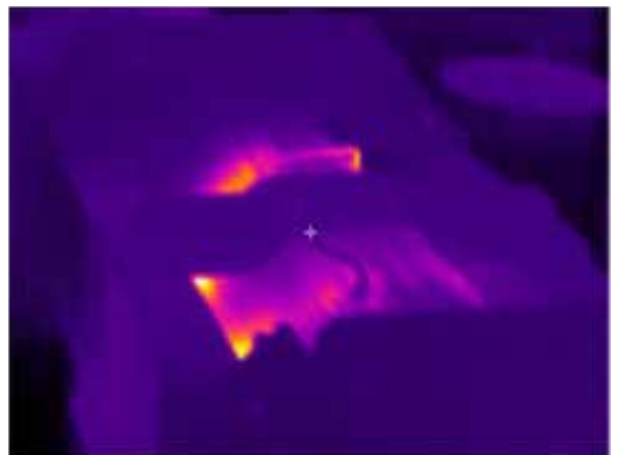

Figura 20. Yugo superior (punto caliente alcanza $102^{\circ} \mathrm{C}$ en los entre hierros del núcleo) Fuente: elaboración propia

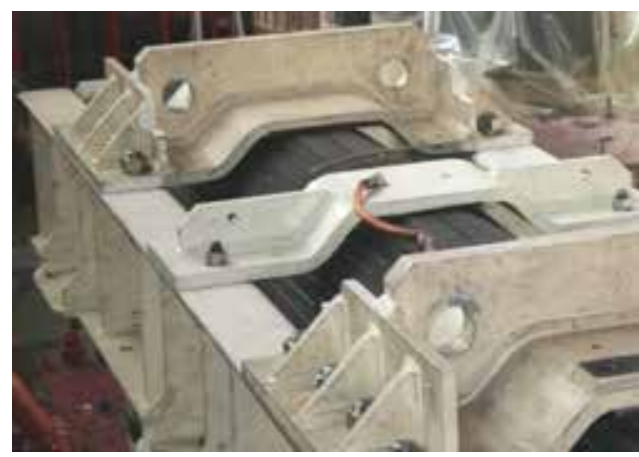

Figura 21. Yugo superior

Fuente: elaboración propia
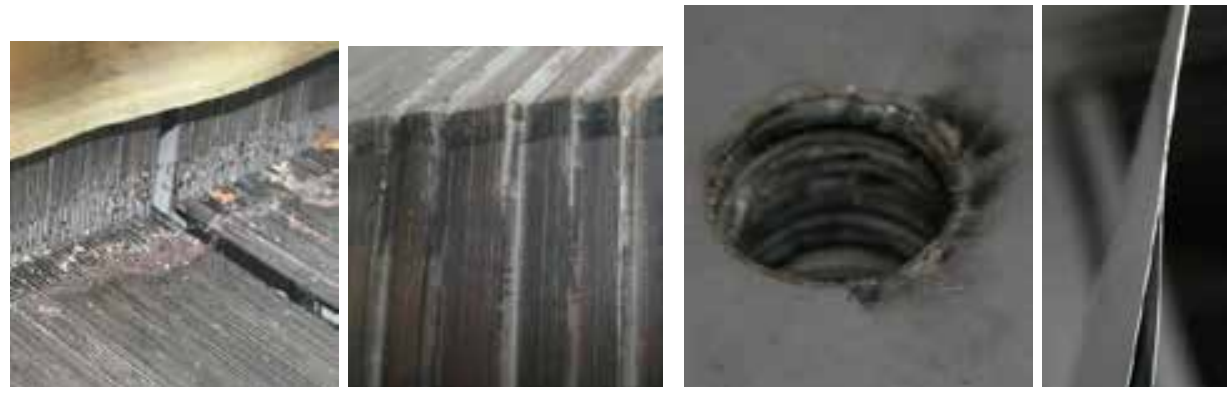

Figura 22. Hallazgos: Traslapes y perforaciones en corto, láminas soldadas en sus extremos Fuente: elaboración propia

Debido al estado del núcleo, este se cambió en su totalidad y se reemplazó el tipo de corte de SSL (Single Step Lap) a MSL (Multi Step Lap) con láminas completas para los yugos superior e inferior, como se presenta en la figuras 23 y 24 . 


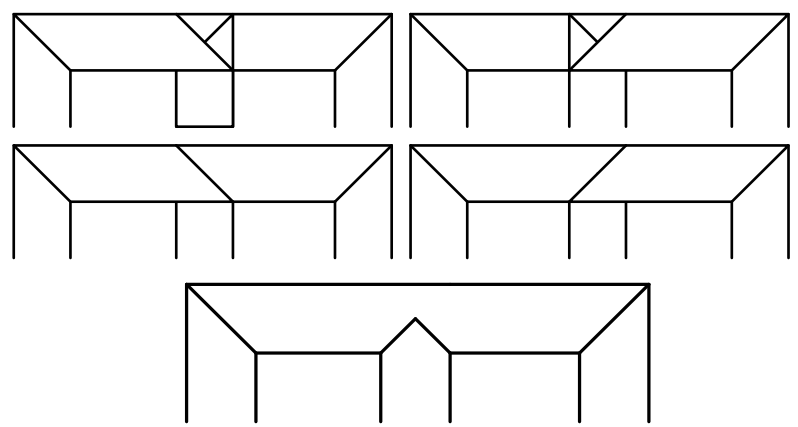

Figura 23. Cierre de yugos núcleo original (superior) y núcleo nuevo (inferior). Fuente: elaboración propia

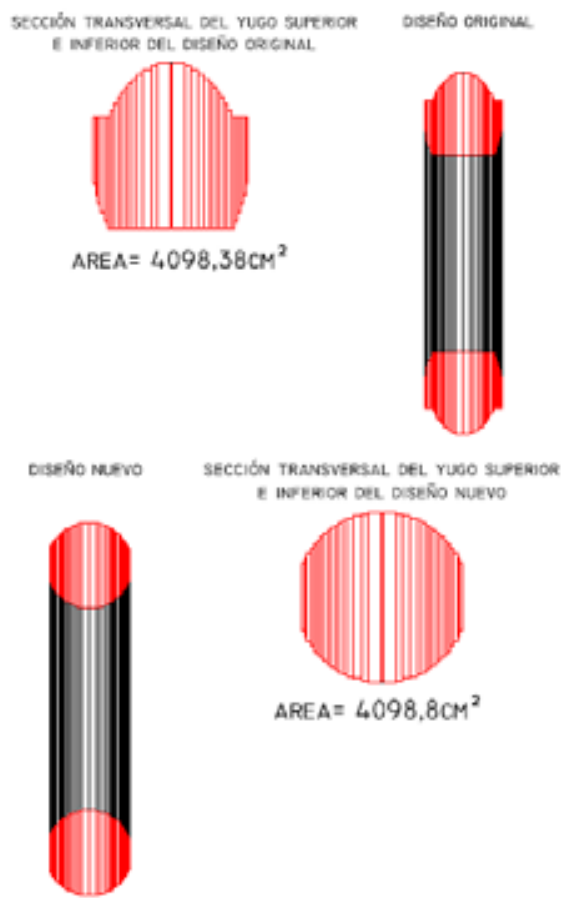

Figura 24. Cambio del sistema de cierre de los yugos Fuente: elaboración propia

El tipo de lámina utilizada en el nuevo diseño fue $\mathrm{M} 4 \mathrm{de} 0,27 \mathrm{~mm}$; el peso original del núcleo era $34542 \mathrm{~kg}$ y con láminas nuevas fue de $35947 \mathrm{~kg}$; la sección transversal de las columnas se conservó con respecto al diseño original. No se evidenciaron puntos calientes en la prueba de inspección termográfica con el núcleo nuevo, como se presenta en las figuras 25 a 28. 


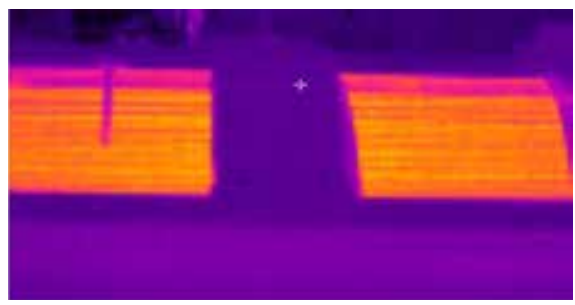

Figura 25. Termografía final sin puntos calientes Fuente: elaboración propia

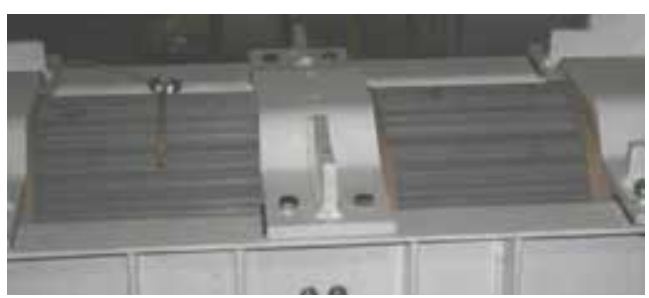

Figura 26. Yugo superior nuevo

Fuente: elaboración propia

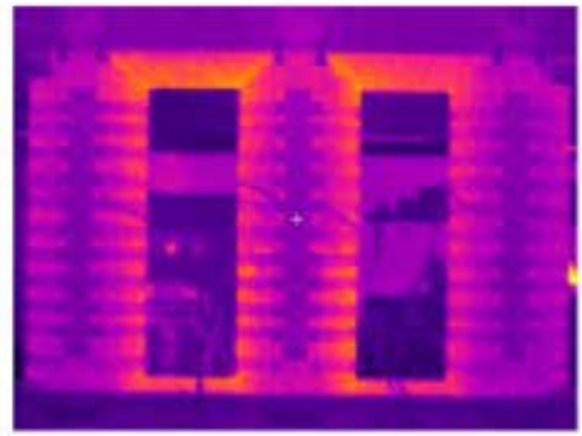

Figura 27. Termografía final sin puntos calientes Fuente: elaboración propia

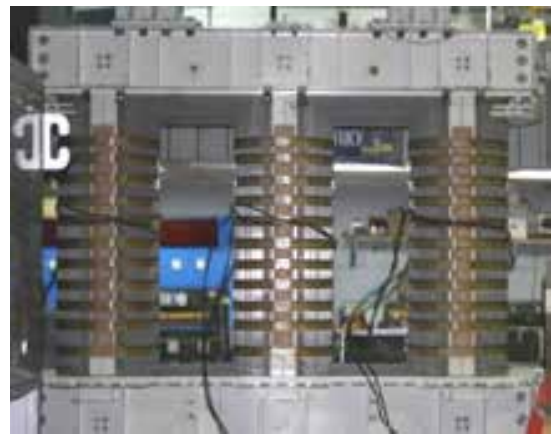

Figura 28. Vista general del núcleo Fuente: elaboración propia 
Se observó una disminución de las pérdidas en vacío cercanas al 20 \% y disminución de la corriente de vacío al 50 \% como indica la figura 29.

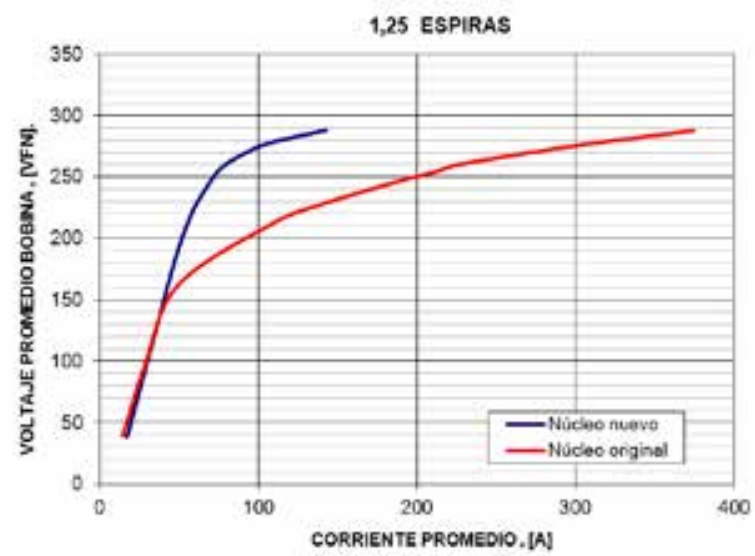

Figura 29. Comparación del voltaje bobina contra corriente en núcleo original y núcleo nuevo durante prueba de magnetización.

Fuente: elaboración propia

La reducción de las pérdidas de vacío fue de $5,25 \mathrm{~kW}$ que representan un ahorro cercano a los USD 105.000 en un período de 20 años; considerando que las bobinas, bujes y demás accesorios se encontraban en buen estado, y el costo del núcleo nuevo era cercano al $20 \%$ del valor de reposición a nuevo del equipo, se demuestra que la reparación fue muy rentable.

\subsection{CASO 3. Transformador monofásico 40/60 MVA, 220 / 110 / 46 kV}

Un banco trifásico de unidades del año 1978 presentó falla en una unidad. La empresa de energía decidió reparar la unida fallada y reconstruir las otras dos unidades. La falla en las bobinas de la unidad del transformador fue considerable y alcanzó a realizar cortos gran parte del núcleo, como se muestra en la figura 30.
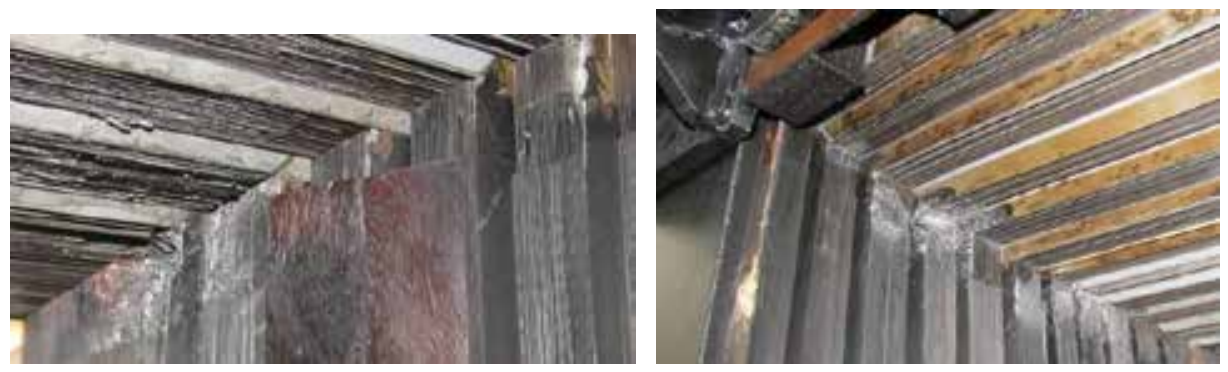

Figura 30. Cortos en el núcleo de unidad fallada.

Fuente: elaboración propia 
Se realizó la prueba de magnetización y medición de pérdidas en los tres núcleos y el núcleo con cortos evidenció puntos calientes en los grupos de láminas de medio núcleo; adicionalmente este presentó un aumento del $10 \%$ en las pérdidas de vacío (tabla 3); a esta unidad se le cambió medio núcleo. Las pruebas finales del núcleo ya reparado evidenciaron una disminución del $5 \%$ de las pérdidas de vacío respecto a las otras dos unidades (tabla 3), y disminución en la temperatura del núcleo nuevo respecto al original, como se observa en las figuras 31 y 32 .
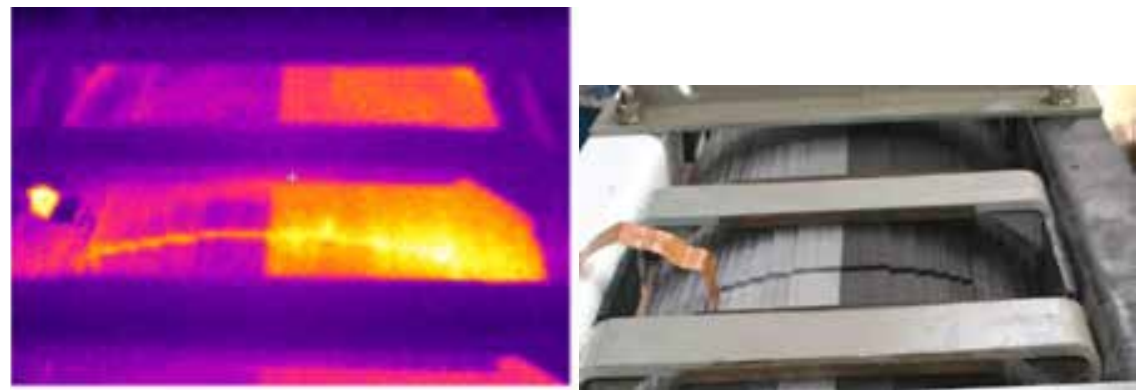

Figura 31. Lado original se calienta $4{ }^{\circ} \mathrm{C}$ por encima de la temperatura del núcleo nuevo Fuente: elaboración propia
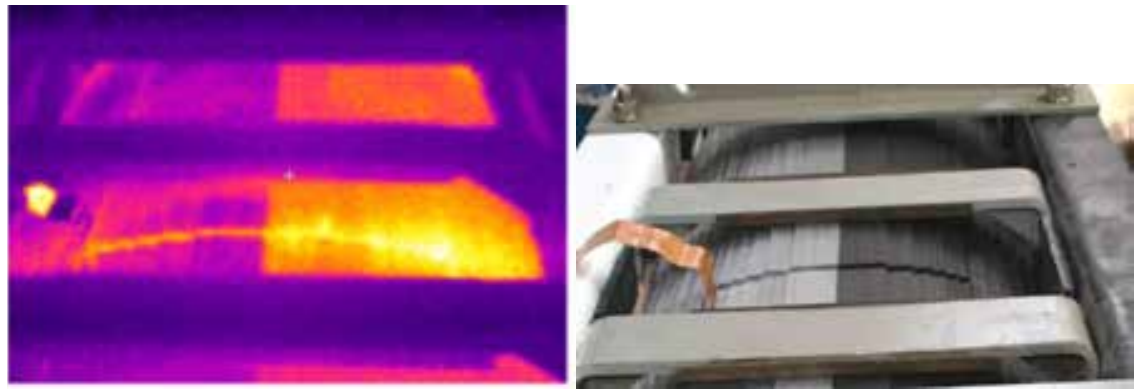

Figura 31. Lado original se calienta $4{ }^{\circ} \mathrm{C}$ por encima de la temperatura del núcleo nuevo Fuente: elaboración propia

El costo total de reconstrucción de este equipo fue de \$ USD 350.000, incluyendo el reaislamiento de todas sus bobinas y el cambio de medio núcleo, el cual fue de $\$$ USD 93.000, comparado con el valor de reposición a nuevo que es de \$USD 550.000 aproximadamente, es decir $60 \%$ del valor del equipo. Estas cifras demuestran que la reparación fue muy rentable. Adicionalmente, las pérdidas de vacío presentaron una disminución de $4 \mathrm{~kW}$, representando un ahorro cercano a los USD 80.000 en un período de 20 años. 


\section{CONCLUSIONES}

La técnica más usada para identificar fallas en el núcleo en el análisis de condición consiste en el análisis de los gases disueltos en el aceite. Esta técnica es muy sensible en un amplio rango para encontrar una inadecuada operación térmica o eléctrica. El hidrógeno y los gases hidrocarbonos son generados debido al rompimiento térmico del aceite mineral que indica en ciertas relaciones la severidad del sobrecalentamiento. La presencia de poco acetileno y un amplio espectro de otros gases, usualmente es el resultado de partes metálicas sobrecalentadas por concentraciones de flujo o corrientes circulantes sin celulosa presente, indicando láminas del núcleo en cortocircuito, cortos entre el núcleo y prensas o partes metálicas; sin embargo, se recomienda realizar una inspección interna a través de un manhole o handhole para comprobar si existen o no cortos en el núcleo.

Las pérdidas de vacío Po y los niveles de ruido de los núcleos de transformadores de potencia son reducidos reemplazando las antiguos uniones de traslape sencillo SSL por uniones con traslapes múltiples MSL debido a que son disminuidas las concentraciones locales de flujo en la región del traslape.

Las reparaciones parciales pueden ser realizadas cuando existen cortos puntuales; en el caso del autotransformador monofásico, estas reparaciones funcionaron en los puntos reparados, pero debido al mal corte efectuado desde su fabricación se formaron nuevos cortos en otros puntos.

En el caso de cambio de núcleo en un transformador reparado, la selección del tipo de lámina se realiza partiendo de las pérdidas de vacío garantizadas por el fabricante; seguidamente se realiza la medición del calibre de la lámina y el peso total del núcleo; posteriormente se calcula la densidad de flujo de operación del núcleo y con estos datos se obtienen las pérdidas de vacío por kilogramo y, finalmente, se selecciona la lámina adecuada de acuerdo con la densidad de flujo de operación.

En el caso del transformador trifásico de 104,5 MVA al cambiar el tipo de traslape de SSL a MSL y el tipo de construcción de los yugo superior e inferior de dos láminas a una lámina, se evidenció una disminución de las pérdidas en vacío cercanas al $20 \%$ y disminución de la corriente de vacío cercana al $50 \%$.

Cuando se realiza reparación de un transformador, es recomendable adecuar la conexión del núcleo a tierra a través de un buje auxiliar, de tal forma que ésta se pueda realizar de forma externa posibilitando la prueba de resistencia de aislamiento núcleo contra prensa cuando se requiera realizarla en sitio.

Se resalta la importancia de que las empresas eléctricas verifiquen con los fabricantes el proceso de corte y terminado final del núcleo para evitar problemas durante la operación del transformador. 
Para que el cambio total o parcial del núcleo sea una opción rentable, es necesario emplear láminas con menores pérdidas. Cuando se realiza la reconstrucción total del transformador con cambio de núcleo, si el valor es de máximo el $70 \%$ del valor de reposición a nuevo, la reparación es rentable.

\section{AGRADECIMIENTOS}

Los autores agradecen a los que han permitido, ayudado y colaborado con la obtención de los resultados referidos en el artículo, a Industrias Explorer, a las Empresas Públicas de Medellín EPM y a la Central Hidroeléctrica de Caldas CHEC.

\section{REFERENCIAS}

[1] I. Albana et al., "Behaviour of transformers cores with multi-step-lap joints". presentado en Intl. Conf. ICEM 2000, Espoo Finland, 2000.

[2] M. J Heathcote, "J\&P Transformer Book" Newness Editorial, Twelfth Edition, pp. 41-53, Great Britain, 1998.

[3] I. Albana, "Behavior of transformer cores with multistep-lap joints". Power Engineering Letters IEEE. Power Engineering Review, presentado en Intl. Conf. Energy Integration in Northeast Asia. Pp. 43-47, Rusia, 2002

[4] Cogent Power Inc. "Multi-Step Lap Construction, A competitive Tool Offering Low Core Loss and Noise Level". [En línea], acceso 30 de octubre, 2013; Disponible: http://www. cogentpowerinc.com, 2010.

[5] CG Power Systems Belgium NV. "Power transformers, your partner in energy". [En línea], acceso 25 de octubre, 2013; Disponible: http://www.belgium@cgglobal.com, 2008.

[6] IEEE Guide for the Interpretation of Gases Generated in Oil-Immersed Transformers. IEEE Std C57.104, 2008

[7] E. E. Staff del M. I. T. “Circuitos Magnéticos y transformadores”. Pp. 44, 161-163, Editorial Reverté. Buenos Aires, 1981.

[8] ThyssenKrupp Electrical Steel India. “Grain Oriented Electrical Steel PowerCore ${ }^{\mathrm{TM}}$ Insulation Types”. [En línea], acceso 2 de noviembre, 2013. Disponible: http://www.tkesindia.com/, 2010

[9] IEC Magnetic materials -Part 1-1. Classification - Surface insulations of electrical steel sheet, strip and laminations, CEI/IEC 60404-1-1, 2004.

[10] IEC Magnetic materials -Part 11. Method of test for the determination of surface insulation resistance of magnetic sheet and strip, CEI/IEC 60404-11, 2012

[11] IEEE Guide for Diagnostic Field Testing of Fluid-Filled Power Transformers, Regulators and Reactors. IEEE Std C.57-152, 2013. 